\title{
Characterization of polymorphic microsatellite loci in the neotropical plant-ant Allomerus decemarticulatus (Formicidae: Myrmicinae) and multiplexing with other microsatellites from the ant subfamily Myrmicinae
}

\author{
Pierre-Jean G. MALÉ ${ }^{1,2}$, Anne LOISEAU ${ }^{3}$, Arnaud ESTOUP ${ }^{3}$, Angélique QUILICHINI $^{4,5}$ and JÉrôMe ORIVEL ${ }^{1,2,4}$ \\ ${ }^{1}$ Université de Toulouse, Laboratoire Evolution \& Diversité Biologique (EDB), UMR 5174, 118 route de Narbonne, \\ 31062 Toulouse cedex 9, France; e-mails: pjmale@cict.fr, orivel@cict.fr \\ ${ }^{2}$ CNRS, EDB (Laboratoire Evolution et Diversité Biologique), F-31062 Toulouse, France \\ ${ }^{3}$ INRA, UMR CBGP (INRA / IRD / Cirad / Montpellier SupAgro), Campus international de Baillarguet, CS 30016, F-34988 \\ Montferrier-sur-Lez cedex, France; e-mails: loiseau@supagro.inra.fr, estoup@supagro.inra.fr \\ ${ }^{4}$ CNRS, UMR Ecologie des Forêts de Guyane, Campus Agronomique, F-97379 Kourou cedex, France; \\ e-mail: Angelique.Quilichini@ecofog.gf \\ ${ }_{5}^{5}$ Jardin Botanique Henri Gaussen, 39 allées Jules Guesde, F-31062 Toulouse, France
}

Key words. Hymenoptera, Formicidae, Myrmicinae, Allomerus, ant-plant mutualism, microsatellites, plant-ant

\begin{abstract}
Five polymorphic microsatellite loci of the arboreal ant Allomerus decemarticulatus (Myrmicinae) were isolated and characterized. The amplification and polymorphism of seven additional microsatellite loci, previously developed for the ant species A. octoarticulatus and Wasmannia auropunctata, were also tested and the amplification conditions necessary for genotyping the complete set of 12 multiplexed markers in A. decemarticulatus determined. The number of alleles per locus ranged from three to 15 and observed heterozygosity varied from 0.09 to 0.95 . Cross-species amplification of these loci was also successfully achieved in additional species of the same ant subfamily, Myrmicinae. This set of microsatellite markers will be used in studies on the mating system and population genetic structure of Myrmicinae in general and A. decemarticulatus in particular.
\end{abstract}

\section{INTRODUCTION}

Allomerus decemarticulatus (Hymenoptera: Myrmicinae) is a neotropical obligate plant-ant. In French Guiana, it is strictly associated with the myrmecophytic understory treelet Hirtella physophora (Chrysobalanaceae) via a mutualistic relationship (Solano et al., 2003; Grangier et al., 2009). Its occurrence is thus restricted to areas where $H$. physophora is present. Populations of $H$. physophora are patchily distributed, even at small geographic scales (Solano et al., 2003), and the population genetic structure of $A$. decemarticulatus is expected to reflect that of its host. A set of polymorphic microsatellite loci for A. decemarticulatus was developed and characterized that can be multiplexed with microsatellite loci previously developed either in a congeneric species or a species from a different tribe of the same subfamily. This set of microsatellite markers will be used in studies on the mating system and population genetic structure of $A$. decemarticulatus.

\section{MATERIAL AND METHODS}

Total genomic DNA was extracted from four adult males using the DNeasy Blood \& Tissue Kit (QIAGEN, Courtaboeuf, France). Extracts were mixed and concentrated using a SpeedVac Concentrator RC1010 (Jouan, Saint-Herblain, France) and re-suspended in ultrapure water to obtain a concentration of $65 \mathrm{ng} / \mu \mathrm{L}$. RsaI-digested DNA fragments from 600 to $1000 \mathrm{~kb}$ in size were extracted from the gel, after electrophoresis, using QIAquick Gel Extraction Kit (QIAGEN). These fragments were ligated to Rsa-21 and Rsa-25 linkers [Rsa-21 (5'-3') CTCTTGCTTACGCGTGGACTA; Rsa-25 (5'-3') phosphateTAGTCCACGCGTAAGCAAGAG] for PCR amplification with Rsa-21 primers. The PCR products were purified with the
QIAquick PCR Purification Kit (QIAGEN). Enrichment was conducted using Streptavidin MagneSphere ${ }^{\circledR}$ Paramagnetic Particles Kit (Promega, Carbonnières-les-Bains, France) and a mixture of the biotinylated oligonucleotide probes (TC) $)_{10}$ and $(\mathrm{TG})_{10}$. Enriched DNA was then amplified using Rsa-21 primers, purified using the QIAquick PCR Purification Kit (QIAGEN) and ligated into pGEM-T vectors using the pGEM-T Easy Vector system II kit (Promega) following the manufacturer's protocol. A total of 1152 white transformant clones were PCR screened using (TC) $)_{10}$ or $(\mathrm{TG})_{10}$ probes with T7 and SP6 primers. One hundred and fifty-two clones gave a positive signal and the inserted DNA fragment was subsequently sequenced. Thirty-six primer pairs were designed using the software Primer Designer v 2.0 (Scientific \& Educational Software 1990, 91). PCR amplifications were carried out in a $15 \mu \mathrm{L}$ final volume containing $1 \times$ QIAGEN Multiplex Master Mix, $0.5 \times$ Q-Solution, $2 \mu \mathrm{M}$ of each primer, and $2 \mu \mathrm{L}$ of genomic DNA. The thermal profile started with an initial denaturation at $95^{\circ} \mathrm{C}$ $(15 \mathrm{~min})$, followed by 35 cycles of denaturation at $94^{\circ} \mathrm{C}(30 \mathrm{~s})$, annealing at $57^{\circ} \mathrm{C}(1 \mathrm{~min} 30 \mathrm{~s})$ and extension at $72^{\circ} \mathrm{C}(1 \mathrm{~min})$ and ended with a final extension at $60^{\circ} \mathrm{C}$ for $30 \mathrm{~min}$. PCR products were sized using electrophoresis on a $1.5 \%$ agarose gel.

Loci for which amplifications produced only one DNA band corresponding to the predicted size of the PCR product were then amplified following the protocol described above but with forward primers fluorophore-labelled on the 5' end and nonlabelled reverse primers. These loci were finally sized using an ABI 3730 sequencer (Applied Biosystems, Courtaboeuf, France) with the 500 LIZ $^{\mathrm{TM}}$ GeneScan $^{\mathrm{TM}}$ size standard and GENEMAPPER $^{\mathrm{TM}} 4.0$ software. Levels of polymorphism were estimated by genotyping one worker per colony from 68 colonies derived from two populations. DNA extractions were per- 
TABLE 1. Primer sequences and polymorphism statistics for 12 microsatellite loci in two populations of Allomerus decemarticulatus [ $\mathrm{Ta}$ - annealing temperature; $n$ - number of colonies; $\mathrm{Na}$ - number of alleles studied; $\mathrm{Ho}$ - proportion of heterozygous individuals; $\mathrm{He}$ - expected heterozygosity ; * - significant deviation from Hardy-Weinberg equilibrium after correction for multiple tests (Sequential Bonferroni procedure; $P<0.01$ )].

\begin{tabular}{|c|c|c|c|c|c|c|c|c|c|c|c|c|c|c|}
\hline \multirow[t]{2}{*}{ Locus } & \multirow{2}{*}{$\begin{array}{l}\text { Repeat motif } \\
\text { (cloned allele) }\end{array}$} & \multirow{2}{*}{$\begin{array}{l}\text { Size } \\
\text { range } \\
(\mathrm{bp})\end{array}$} & \multirow{2}{*}{$5^{\prime}$ dye } & \multicolumn{3}{|c|}{$\begin{array}{l}\text { Petit Saut } \\
(n=46)\end{array}$} & \multicolumn{3}{|c|}{$\begin{array}{l}\text { Nouragues } \\
(n=22)\end{array}$} & \multirow{2}{*}{$\begin{array}{l}T a \\
\left({ }^{\circ} \mathrm{C}\right)\end{array}$} & \multirow{2}{*}{ Primer sequences $\left(5^{\prime}-3^{\prime}\right)$} & \multirow{2}{*}{$\begin{array}{l}\text { Multiplex } \\
\text { markers } \\
\text { set }\end{array}$} & \multirow{2}{*}{$\begin{array}{c}\text { Genbank } \\
\text { accession } \\
\text { numbers }\end{array}$} & \multirow{2}{*}{ Source } \\
\hline & & & & $\mathrm{Na}$ & Ho & $\mathrm{He}$ & $\mathrm{Na}$ & Ho & $\mathrm{He}$ & & & & & \\
\hline Adec-A21 & $(\mathrm{AG})_{22}$ & $356-360$ & $6 \mathrm{FAM}$ & 3 & 0.54 & 0.45 & 3 & 0.68 & 0.52 & 63.8 & $\begin{array}{l}\text { TACCGGACCGAACTACTTGC } \\
\text { ACTCACGCACTCGCCTCAT }\end{array}$ & 2 & GU982709 & This study \\
\hline Adec-A23 & $(\mathrm{GA})_{30}$ & $289-295$ & $6 \mathrm{FAM}$ & 7 & 0.23 & 0.30 & 6 & 0.72 & 0.80 & 64.2 & $\begin{array}{c}\text { ACGAACGCTGCACTTCCA } \\
\text { AGGAGGTGAGAGAGCTTGAGG }\end{array}$ & 1 & GU982710 & This study \\
\hline Adec-A41 & $(\mathrm{AG})_{18}$ & $161-167$ & $6 \mathrm{FAM}$ & 3 & 0.65 & $0.90^{*}$ & 3 & 0.41 & 0.58 & 62.2 & $\begin{array}{l}\text { CGTATCCATAAGGCGCAATC } \\
\text { GTCGTCAAGAAGCAGAGGCA }\end{array}$ & 1 & GU982711 & This study \\
\hline Adec-A49 & $(\mathrm{AG})_{32}$ & $212-226$ & $6 \mathrm{FAM}$ & 8 & 0.73 & 0.64 & 6 & 0.81 & 0.74 & 62.1 & $\begin{array}{l}\text { CGTGCCATCGCGAGAA } \\
\text { CCATTTCTGCCGAGGGT }\end{array}$ & 1 & GU982712 & This study \\
\hline Adec-A60 & $(\mathrm{GA})_{38}$ & $271-283$ & NED & 4 & 0.09 & 0.09 & 3 & 0.64 & 0.55 & 60.9 & $\begin{array}{c}\text { TCGCGCGGAAATCGT } \\
\text { CATCTGTCGTGCACCGTAAT }\end{array}$ & 2 & GU982713 & This study \\
\hline Waur8 $\Omega$ & $(\mathrm{GA})_{15}$ & $273-313$ & PET & 7 & 0.76 & 0.82 & 12 & 0.82 & 0.89 & 57 & $\begin{array}{l}\text { GTAGTTGGCGAGACCGGATG } \\
\text { CTCCAGCTGTGGTTCCGATG }\end{array}$ & 1 & FJ970016 & $\begin{array}{c}\text { Molecular } \\
\text { Ecology } \\
\text { Resources } \\
\text { Primer } \\
\text { Development } \\
\text { Consortium } \\
\text { et al., } 2009\end{array}$ \\
\hline Waur872 & $(\mathrm{GA})_{12} \mathrm{AA}(\mathrm{GA})_{23}$ & $357-415$ & VIC & 11 & 0.55 & $0.80^{*}$ & 8 & 0.20 & $0.87 *$ & 57 & $\begin{array}{l}\text { CGTGCTATCCTCGACGAAGT } \\
\text { GCCGACAGTACAACAAACAGC }\end{array}$ & 2 & FJ970023 & $\begin{array}{c}\text { Molecular } \\
\text { Ecology } \\
\text { Resources } \\
\text { Primer } \\
\text { Development } \\
\text { Consortium } \\
\text { et al., } 2009\end{array}$ \\
\hline Waur813 & $(\mathrm{GA})_{17}$ & $106-145$ & NED & 15 & 0.76 & 0.85 & 10 & 0.95 & 0.89 & $57^{C}$ & $\begin{array}{l}\text { GTCCAAGGATGAATGTATTCAC } \\
\text { GCCGCTAGAAGAAGAATGAC }\end{array}$ & 2 & FJ970021 & $\begin{array}{c}\text { Molecular } \\
\text { Ecology } \\
\text { Resources } \\
\text { Primer } \\
\text { Development } \\
\text { Consortium } \\
\text { et al., } 2009\end{array}$ \\
\hline Waur730 & (CT)18 & $182-209$ & VIC & 7 & 0.39 & 0.42 & 9 & 0.80 & 0.87 & 57 & $\begin{array}{l}\text { GAATGAGCGAAGCGTGTTAC } \\
\text { GAGATTCTCGACGCGTATGA }\end{array}$ & 1 & AY779642 & $\begin{array}{l}\text { Fournier } \\
\text { et al., } 2005\end{array}$ \\
\hline Waur225 & $(\mathrm{CA})_{10}$ & $240-290$ & $\mathrm{VIC}$ & 5 & 0.20 & $0.34 *$ & 8 & 0.67 & 0.75 & 63 & $\begin{array}{l}\text { GTGCGCAGACATAGATAAGG } \\
\text { TGAATAGCTGCGACTCTACG }\end{array}$ & 2 & AY779634 & $\begin{array}{l}\text { Fournier } \\
\text { et al., } 2005\end{array}$ \\
\hline Ad166 & $(\mathrm{TG})_{11}$ & $161-176$ & NED & 3 & 0.32 & $0.51 *$ & 11 & 0.77 & 0.85 & 53.5 & $\begin{array}{l}\text { GGTCCTTTGAGCAACTTAGC } \\
\text { CTGATCGCAATAGAGCAATG }\end{array}$ & 1 & DQ139976 & $\begin{array}{c}\text { Debout } \\
\text { et al., } 2006\end{array}$ \\
\hline Ad040 & $(\mathrm{TG})_{16}(\mathrm{CG})_{5}(\mathrm{CA})_{3}$ & ${ }_{3} 301-331$ & VIC & 9 & 0.39 & $0.85^{*}$ & 14 & 0.75 & 0.88 & 50.4 & $\begin{array}{l}\text { GAAAGACAGATCGCTTCATC } \\
\text { GCCGATATTACTTCATTCAG }\end{array}$ & 2 & DQ139967 & $\begin{array}{c}\text { Debout } \\
\text { et al., } 2006\end{array}$ \\
\hline
\end{tabular}

formed using 10\% Chelex (BioRad, Marnes-la-Coquette, France) solution. Level of polymorphism and deviation from Hardy-Weinberg equilibrium were estimated for each locus using GENEPOP 4.0 (Raymond \& Rousset, 1995; Rousset, 2008). Correction for multiple tests was performed when appropriate (Sequential Bonferroni procedure).

\section{RESULTS AND DISCUSSION}

Fourteen PCR products gave discrete bands of the predicted size on agarose gels. All of these loci were unambiguously sized using the sequencer. Among them, five loci were polymorphic. Individual loci had 3 to 8 alleles and observed heterozygosity ranged between 0.09 and 0.95 (Table 1). Five loci departed significantly from Hardy-Weinberg equilibrium $(P<0.01$, Table 1). It is likely this can be accounted for by a high level of inbreeding in populations of $A$. decemarticulatus due to the clumped distribution of $H$. physophora.

Sequences of the five microsatellite loci are deposited in the GenBank database (see Table 1 for accession numbers). All loci were successfully cross-amplified in at least one species of three in the same genus (A. octoarticulatus, A. septemarticulatus, and A. dentatus), one species of the same tribe (Monomorium subopacum), and one species in a different tribe but the same subfamily (Wasmannia auropunctata) (Table 2).

To increase the number of loci available for mating system and population genetic studies in A. decemarticulatus, the coamplification of the five above markers plus seven microsatellite loci previously developed for two ant species from the same subfamily (two loci from $A$. octoarticulatus and five from $W$. auropunctata; Fournier et al., 2005; Debout et al., 2006; Molecular Ecology Resources Primer Development Consortium 
TABLE 2. Cross-species PCR tests for five Allomerus decemarticulatus microsatellite loci in five ant species (A. octoarticulatus, A. septemarticulatus, A. dentatus, Monomorium subopacum, and Wasmannia auropunctata). The number of alleles and their size range (in parentheses) are based on genotyping $n$ individuals of each species. Amplification failure is indicated by a dash.

\begin{tabular}{lccccc}
\hline Locus & $\begin{array}{c}\text { A. octoarticulatus } \\
(n=18)\end{array}$ & $\begin{array}{c}\text { A. septemarticulatus } \\
(n=3)\end{array}$ & $\begin{array}{c}\text { A. dentatus } \\
(n=1)\end{array}$ & $\begin{array}{c}\text { M. subopacum } \\
(n=1)\end{array}$ & $\begin{array}{c}\text { W. auropunctata } \\
(n=5)\end{array}$ \\
\hline Adec-A21 & $5(351-360)$ & $3(350-358)$ & $1(358)$ & $1(358)$ & $3(356-360)$ \\
Adec-A23 & $6(275-290)$ & $3(268-290)$ & $2(300-304)$ & $1(289)$ & $3(291-307$ \\
Adec-A41 & $44(161-171)$ & $4(161-169)$ & $2(165-175)$ & $2(161-165)$ & $4(162-184)$ \\
Adec-A49 & $5(212-226)$ & $2(214-222)$ & $2(214-228)$ & - & - \\
Adec-A60 & $6(214-226)$ & $4(271-279)$ & $2(272-284)$ & $2(272-284)$ & $4(273-279)$ \\
\hline
\end{tabular}

et al., 2009) was optimized. These seven markers were chosen based on their putative polymorphism in $A$. decemarticulatus. Forward primers were fluorescently labelled according to expected size ranges in order to allow typing of all 12 loci in a single electrophoresis run. Two sets of six primer pairs were each designed according to annealing temperature using Multiplex Manager 1.1 (Holleley \& Geerts, 2009). This complete set of markers will be used in studies on the dispersal and mating biology of an ecologically and evolutionarily important antplant mutualism.

ACKNOWLEDGEMENTS. We are grateful to R. Boulay for providing the sample of Monomorium subopacum, T. Izzo for samples of Allomerus septemarticulatus and A. dentatus, K.G. Dexter for editing the manuscript and the Laboratoire Environnement de Petit Saut for furnishing logistical help. This study was funded by a research program of the French Agence Nationale de la Recherche (research agreement $\mathrm{n}^{\circ}$ ANR-06JCJC-0109-01), the ESF-EUROCORES/TECT/BIOCONTRACT program, the Programme Amazonie II of the French Centre National de la Recherche Scientifique and a Nouragues research grant from CNRS.

\section{REFERENCES}

Debout G.D.G., Pereyra R., Emerson B.C. \& Yu D.W. 2006: Characterization of polymorphic microsatellites in the castration parasite plant-ant Allomerus octoarticulatus cf. demerarae (Formicidae: Myrmicinae). Mol. Ecol. Notes 6: 182-184.

Fournier D., Foucaud J., Loiseau A., Cros-Arteil S., Jourdan H., Orivel J., Le Breton J., Chazeau J., Dejean A., Keller L. \& Estoup A. 2005: Characterization and PCR multiplexing of polymorphic microsatellite loci for the invasive ant Wasmannia auropunctata. Mol. Ecol. Notes 5: 239-242.

Grangier J., Dejean A., Malé P.-J.G., Solano P.-J. \& Orivel J. 2009: Mechanisms driving the specificity of a myrmecophyte-ant association. Biol. J. Linn. Soc. 97: 90-97.

Holleley C.E. \& GeERTS P. 2009: Multiplex Manager 1.0: a cross-platform computer program that plans and optimizes multiplex PCR. Biotechniques 46: 511-517.

Molecular Ecology Resources Primer Development Consortium, Almány G.R., De Arruda M.P., Arthofer W., Atallah Z.K., Beissinger S.R., Berumen M.L., Bogdanowicz S.M., Brown S.D., Bruford M.W., Burdine C., Busch J.W., CAmpbell N.R., Carey D., Carstens B.C., Chu K.H., Cubeta
M.A., Cuda J.P., Cui Z.X., Datnoff L.E., Davila J.A., Davis E.S., Davis R.M., Diekmann O.E., Eizirik E., Fargallo J.A., Fernandes F., Fukuda H., Gale L.R., Gallagher E., Gao Y.Q., Girard P., Godhe A., Goncalves E.C., Gouveia L., Grajczyk A.M., Grose M.J., Gu Z.F., Hallden C., Harnstrom K., Hemmingsen A.H., Holmes G., Huang C.H., Huang C.C., Hudman S.P., Jones G.P., Kanetis L., Karunasagar I., Karunasagar I., Keyghobadi N., Klosterman S.J., Klug P.E., Кoch J., Koopman M.M., Koppler K., Koshimizu E., Krumbock S., Kubisiak T., Landis J.B., Lasta M.L., Lee C.Y., Li Q.Q., Li S.H., Lin R.C., Liu M., Liu N., LiU W.C., Liu Y., Loiseau A., Luan W.S., Maruthachalam K.K., McCormick H.M., Mellick R., Monnahan P.J., MorielleVersute E., Murray T.E., Narum S.R., Neufeld K., de Nova P.J.G., Олiambo P.S., Окамоto N., Оthman A.S., Overholt W.A., Pardini R., Paterson I.G., Patty O.A., Paxton R.J., Planes S., Porter C., Pratchett M.S., Puttker T., Rasic G., Rasool B., Rey O., Riegler M., Riehl C., Roberts J.M.K., Roberts P.D., Rochel E., Roe K.J., Rossetto M., Ruzzante D.E., Sakamoto T., Saravanan V., Sarturi C.R., Schmidt A., Schneider M.P.C., Schuler H., Serb J.M., Serrao E.T.A., Shi Y.H., Silva A., Sin Y.W., Sommer S., Stauffer C., Strussmann C.A., Subbarao K.V., Syms C., Tan F., Tejedor E.D., Thorrold S.R., Trigiano R.N., Trucco M.I., Tsuchiya-Jerep M.T.N., Vergara P., van de Vliet M.S., Wadl P.A., Wang A.M., Wang H.X., Wang R.X., Wang X.W., Wang Y., Weeks A.R., Wei F.W., Werner W.J., Wiley E.O., Williams D.A., Wilkins R.J., Wisely S.M., With K.A., Wu D.H., YaO C.T., Yau C., Yeap B.K., Zhai B.P., Zhan X.J., Zhang G.Y., Zhang S.Y., Zhao R. \& Zhu L.F. 2009: Permanent genetic resources added to molecular ecology resources database 1 May 2009-31 July 2009. Mol. Ecol. Resour. 9: 1460-1466.

RAYMOND M. \& Rousset F. 1995: GENEPOP (version 1.2): population genetics software for exact test and ecumenism. $J$. Hered. 86: 248-249.

Rousset F. 2008: Genepop'007: a complete reimplementation of the Genepop software for Windows and Linux. Mol. Ecol. Resour. 8: 103-106.

Solano P.-J., Durou S., Corbara B., Quilichini A., Cerdan P., Belin-Dupoux M., Delabie J.H.C. \& Dejean A. 2003: Myrmecophytes of the understory of French Guianian rainforests: their distribution and their associated ants. Sociobiology 41: 605-614.

Received March 30, 2010; revised and accepted May 10, 2010 